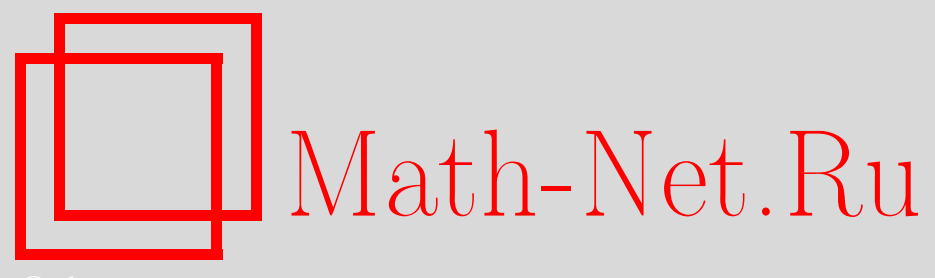

А. Н. Тимашёв, Об асимптотических разложениях для чисел Стирлинга первого и второго рода, Дискрет. матем., 1998, том 10, выпуск 3, 148-159

DOI: https://doi.org/10.4213/dm440

Использование Общероссийского математического портала Math-Net.Ru подразумевает, что вы прочитали и согласны с пользовательским соглашением http: //www.mathnet.ru/rus/agreement

Параметры загрузки:

IP : 54.224 .135 .184

26 апреля 2023 г., 09:11:23

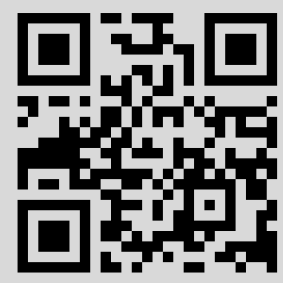




\title{
Об асимптотических разложениях для чисел Стирлинга первого и второго рода
}

\author{
(c) 1998 г. А. Н. Тимашев
}

Рассматривается задача асимптотической оценки чисел Стирлинга первого рода $s(n, N)$ и второго рода $\sigma(n, N)$ при условии, что $n, N \rightarrow \infty$ так, что

$$
1<\alpha_{0} \leqslant \alpha=\frac{n}{N} \leqslant \alpha_{1}<\infty,
$$

где $\alpha_{0}, \alpha_{1}$ - постоянные. Путем использования метода перевала показано, что коэффициенты при отрицательных степенях вида $N^{-m}, m=1,2, \ldots$, в асимптотических разложениях чисел $s(n, N)$ и $\sigma(n, N)$ по степеням $N^{-1}$ при этом условии определяются из представления в виде суммы степенного ряда некоторой функции, зависящей от решения заданного нелинейного дифференциального уравнения первого порядка с известным начальным условием. $\mathrm{C}$ использованием этих результатов найдены линейные рекуррентные соотношения в комплексной области, которым удовлетворяют указанные коэффициенты. В качестве следствий из этих соотношений приводятся явные формулы для коэффициента при $N^{-1}$.

\section{1. Числа Стирлинга первого рода}

Рассмотрим задачу асимптотической оценки чисел Стирлинга первого рода $s(n, N)$ при условии, что $n, N \rightarrow \infty$ так, что

$$
1<\alpha_{0} \leqslant \alpha=\frac{n}{N} \leqslant \alpha_{1}<\infty,
$$

где $\alpha_{0}, \alpha_{1}$ - постоянные.

Вопросам получения асимптотических оценок для чисел Стирлинга первого рода при различных предположениях об изменении параметров $n, N$ посвящено к настоящему времени много работ (см., например, [1], с. 149-161). В частности, в работе Гуда [2] впервые получен главный член асимптотического разложения для чисел $s(n, N)$ и $\alpha=n / N$, а также от единственного корня $z=z_{0} \in(0,1)$ уравнения

$$
\frac{z}{(z-1) \ln (1-z)}=\alpha>1
$$

В данной работе путем использования метода перевала показано, что коэффициенты при отрицательных степенях вида $N^{-m}, m=1,2, \ldots$, в асимптотическом разложении чисел $s(n, N)$ при условии (1) определяются из представления в виде суммы степенного ряда некоторой функции, зависящей от решения заданного 
дифференциального уравнения первого порядка с известным начальным условием. Используя этот результат, найдены линейные рекуррентные соотношения в комплексной области, которым удовлетворяют указанные коэффициенты. В качестве следствия из этих соотношений приводится явная формула для коэффициента при $N^{-1}$.

Для получения искомых рекуррентных соотношений будем исходить из известного равенства [1]

$$
|s(n, N)|=\frac{n !}{2 \pi i N !} \oint \frac{(-\ln (1-z))^{N}}{z^{n+1}} d z .
$$

Интегрирование в (3) осуществляется по окружности радиуса, меньшего единицы, с центром в нуле, пробегаемой в положительном направлении. Для логарифма выбирается та непрерывная ветвь, которая принимает вещественные значения на вещественной положительной полуоси.

Формулу (3) можно представить в виде

$$
|s(n, N)|=\frac{n !}{2 \pi i N !} \oint \varphi(z) \exp (N \omega(z)) d z
$$

где

$$
\varphi(z)=\frac{1}{z}
$$

и при $0<|z|<1$

$$
\omega(z)=\ln (-\ln (1-z))-\alpha \ln z,
$$

если выполнено условие (1).

Из равенства $\omega^{\prime}(z)=0$ получаем, что $z=z_{0} \in(0,1)-$ корень уравнения (2). Такой корень существует и единствен, поскольку функция

$$
q(z)=\frac{z}{(z-1) \ln (1-z)}
$$

непрерывна и строго возрастает от 1 до $\infty$ при возрастании $z$ от 0 до 1. При этом $z_{0} \rightarrow 0+$ при $\alpha \rightarrow 1+$ и $z_{0} \rightarrow 0-$ при $\alpha \rightarrow \infty$. Кроме того, при $\alpha>1$

$$
-\alpha^{-1}+1<z_{0}
$$

поскольку

$$
q\left(z_{0}\right)=\alpha>q\left(-\alpha^{-1}+1\right)=\frac{\alpha-1}{\ln \alpha} .
$$

Будем интегрировать в (4) по окружности $|z|=z_{0}$ (пробегаемой в положительном направлении). На этой окружности находится точка перевала $z=z_{0}$ функции $\omega$, для которой $\omega^{\prime}\left(z_{0}\right)=0$ и

$$
\omega^{\prime \prime}\left(z_{0}\right)=\frac{\alpha\left(\alpha z_{0}-\alpha+1\right)}{z_{0}^{2}\left(1-z_{0}\right)}>0 .
$$

Таким образом, точка $z=z_{0}$ является точкой перевала первого порядка этой функции, причем из условий

$$
|z|=z_{0}, \quad z \neq z_{0}
$$


следует, что

$$
\operatorname{Re} \omega(z)<\omega\left(z_{0}\right)=\ln \left(-\ln \left(1-z_{0}\right)\right)-\alpha \ln z_{0} .
$$

Кроме того, функции $\varphi$ и $\omega$, определяемые из (5) и (6), аналитичны в некоторой области (круговом кольце), содержащей контур интегрирования в (4) (с разрезом по отрицательной вещественной полуоси). Поэтому при условии (1) и $n, N \rightarrow \infty$ справедливо асимптотическое разложение (см. [3], с. 66)

$$
\frac{1}{2 \pi i} \oint_{|z|=z_{0}} \varphi(z) \exp (N \omega(z)) d z \sim \frac{1}{2 \pi i} \sqrt{\frac{\pi}{N}} \exp \left(N \omega\left(z_{0}\right)\right) \sum_{m=0}^{\infty} \frac{(2 m) !}{m ! 2^{2 m}} \frac{a_{2 m}}{n^{m}}
$$

где с учетом (7)

$$
a_{0}=i \varphi\left(z_{0}\right) \sqrt{\frac{2}{\omega^{\prime \prime}\left(z_{0}\right)}}=i \sqrt{\frac{2\left(1-z_{0}\right)}{\alpha\left(\alpha z_{0}-\alpha+1\right)}}
$$

и коэффициенты $a_{r}, r=1,2, \ldots$, определяются из соотношения

$$
\sum_{r=0}^{\infty} a_{r} u^{r}=\varphi\left(z_{0}+\psi(u)\right) \psi^{\prime}(u)
$$

а функция $\psi(u)$ удовлетворяет условиям

$$
\begin{aligned}
\omega\left(z_{0}+\psi(u)\right) & =\omega\left(z_{0}\right)-u^{2} \\
\psi(0)=0, \quad \psi^{\prime}(0) & =i \sqrt{\frac{2}{\omega^{\prime \prime}\left(z_{0}\right)}}=a_{0} z_{0}=i z_{0} \sqrt{\frac{2\left(1-z_{0}\right)}{\alpha\left(\alpha z_{0}-\alpha+1\right)}} .
\end{aligned}
$$

Заметим, что если в асимптотическом разложении (8) ограничиться главным членом, то получим, что при условии (1) и $n, N \rightarrow \infty$

$$
|s(n, N)|=\frac{n !}{N !} \sqrt{\frac{1-z_{0}}{2 \pi n\left(\alpha z_{0}-\alpha+1\right)}} \frac{\left(-\ln \left(1-z_{0}\right)\right)^{N}}{z_{0}^{n}}\left(1+O\left(\frac{1}{N}\right)\right) .
$$

Равенство (13) - это известная формула Гуда [2].

Заметим также, что из условия (1) следует, что асимптотическое разложение (8) (а потому и оценка (13)) справедливо равномерно относительно $\alpha \in\left[\alpha_{0}, \alpha_{1}\right]$.

Теорема 1. Пусть $n, N \rightarrow \infty$ так, что выполнено условие (1). Тогда равномерно относительно $\alpha \in\left[\alpha_{0}, \alpha_{1}\right]$ справедливо асимптотическое разложение

$$
|s(n, N)| \sim \frac{n !}{N !} \sqrt{\frac{1-z_{0}}{2 \pi n\left(\alpha z_{0}-\alpha+1\right)}} \frac{\left(-\ln \left(1-z_{0}\right)\right)^{N}}{z_{0}^{n}}\left(1+\sum_{m=1}^{\infty} \frac{(2 m) !}{m ! 2^{2 m}} \frac{x_{m}}{N^{m}}\right),
$$

где $x_{m}=a_{2 m} / a_{0}, m=1,2, \ldots, u$ коэффичиенты $a_{r}, r=0,1, \ldots$, определяются из (9) и равенства

$$
\sum_{r=0}^{\infty} a_{r} u^{r}=\frac{1}{\alpha}\left(\theta^{\prime}(u) / \theta(u)+2 u\right)
$$


а функция $\theta(u)$ является аналитическим в окрестности нуля решением дифференциалвного уравнения

$$
\alpha \theta^{\prime}(u) \theta(u)=\left(e^{\theta(u)}-1\right)\left(\theta^{\prime}(u)+2 u \theta(u)\right),
$$

удовлетворяющим началвному условию

$$
\theta(0)=-\ln \left(1-z_{0}\right)
$$

При этом $z=z_{0}-$ единственный хоренъ уравнения (2), принадлежащий интервалу $(0,1)$.

Доказательство. Формула (14) следует из (4) и (8); коэффициенты $a_{r}, r=0,1, \ldots$, определяются условиями (9)-(12). Покажем, что из этих условий следуют равенства (15) и (16), а также соотношение

$$
\theta(0)=-\ln \left(1-z_{0}\right)
$$

Из (6) и (11) получаем, что при достаточно малых по модулю значениях $u$

$$
\ln \left(-\ln \left(1-z_{0}-\psi(u)\right)\right)-\alpha \ln \left(z_{0}+\psi(u)\right)=\omega\left(z_{0}\right)-u^{2} .
$$

Дифференцируя это равенство по $u$, находим, что функция $\psi$ удовлетворяет дифференциальному уравнению

$$
\ln \left(1-z_{0}-\psi(u)\right)+\frac{\psi^{\prime}(u)\left(z_{0}+\psi(u)\right)}{\left(1-z_{0}-\psi(u)\right)\left(\alpha \psi^{\prime}(u)-2 u \psi(u)-2 u z_{0}\right)}=0 .
$$

Положим

$$
\theta(u)=-\ln \left(1-z_{0}-\psi(u)\right)
$$

тогда из (12) следует, что

$$
\theta(0)=-\ln \left(1-z_{0}\right)
$$

Кроме того,

$$
\exp (\theta(u))=\left(1-z_{0}-\psi(u)\right)^{-1}
$$

и

$$
\theta^{\prime}(u)=\psi^{\prime}(u) \exp (\theta(u))
$$

поэтому предыдущее дифференциальное уравнение можно переписать в виде (16). Функция $\theta(u)$ аналитична в некоторой окрестности нуля как композиция аналитических функций, причем согласно (12)

$$
\theta^{\prime}(0)=\psi^{\prime}(0) \exp (\theta(0))=i z_{0} \sqrt{\frac{2}{\alpha\left(1-z_{0}\right)\left(\alpha z_{0}-\alpha+1\right)}} .
$$

Поскольку согласно (5) и (10)

$$
\sum_{r=0}^{\infty} a_{r} u^{r}=\frac{\psi^{\prime}(u)}{z_{0}+\psi(u)}=\frac{\theta^{\prime}(u)}{\exp (\theta(u))-1}
$$

из (16) следует (15).

Теорема 1 доказана. 
Теорема 2. Коэффициенты $a_{r}, r=0,1, \ldots$, в асимптотическом разложении (14) удовлетворяют линейным рехуррентным соотношениям в комплексной области

$$
\alpha \sum_{r=0}^{k} b_{k-r} a_{r}=(k+1) b_{k+1}+2 b_{k-1}, \quad k=1,2, \ldots,
$$

с начальным условием (9). В равенствах (18) хоэффичиенты $b_{s}, s=0,1, \ldots$, удовлетворяют линейным рекуррентным соотношениям

$$
b_{k}+\frac{1}{k} \sum_{s=1}^{k} s b_{s} c_{k-s}=c_{k}, \quad k=1,2, \ldots,
$$

с начальными условиями.

$$
\begin{aligned}
b_{0} & =\frac{z_{0}}{\alpha\left(1-z_{0}\right)}, \\
b_{1} & =\frac{z_{0} a_{0}}{1-z_{0}} .
\end{aligned}
$$

При этом коэффичиенты $c_{s}$ связаны $c$ коэффициентами $b_{s}, s=0,1, \ldots$, рекуррентными соотношениями

$$
\begin{aligned}
& \sum_{s=1}^{k-1}\left(\left((k+1-s) c_{s}-\alpha s b_{s}\right) b_{k+1-s}+2 b_{k-1-s} c_{s}\right) \\
& +\alpha\left(2 b_{0} b_{k-1}-b_{1} k b_{k}\right)+b_{1} c_{k}=0, \quad k=2,3, \ldots,
\end{aligned}
$$

с началъными условиями

$$
\begin{aligned}
& c_{0}=\frac{z_{0}}{1-z_{0}} \\
& c_{1}=\frac{z_{0} a_{0}}{\left(1-z_{0}\right)^{2}} .
\end{aligned}
$$

Дожазательство. Положим

$$
\theta(u)=\sum_{s=0}^{\infty} b_{s} u^{s}
$$

тогда согласно (15)

$$
\begin{aligned}
\alpha\left(\sum_{r=0}^{\infty} a_{r} u^{r}\right)\left(\sum_{s=0}^{\infty} b_{s} u^{s}\right) & =\theta^{\prime}(u)+2 u \theta(u) \\
& =b_{1}+\sum_{k=1}^{\infty}\left((k+1) b_{k+1}+2 b_{k-1}\right) u^{k}
\end{aligned}
$$

причем

$$
b_{0}=\theta(0)=-\ln \left(1-z_{0}\right)
$$

и согласно (17)

$$
b_{1}=\theta^{\prime}(0)=\alpha a_{0} b_{0} .
$$


Поскольку $z=z_{0}$ - корень уравнения (2), отсюда получаем (20). Перемножая полученные степенные ряды по Коши и приравнивая коэффициенты при $u^{k}, k=$ $1,2, \ldots$, получаем соотношения (18) с начальным условием (9). Далее положим

$$
\exp (\theta(u))-1=\sum_{s=0}^{\infty} c_{s} u^{s}
$$

тогда

$$
\begin{aligned}
& c_{0}=\exp (\theta(0))-1=\frac{z_{0}}{1-z_{0}} \\
& c_{1}=\theta^{\prime}(0) \exp (\theta(0))
\end{aligned}
$$

Согласно (17) отсюда получаем начальные условия (22). Если ввести обозначение

$$
h(u)=\exp (\theta(u))-1
$$

To

$$
h^{\prime}(u)=\theta^{\prime}(u)(h(u)+1),
$$

и поэтому при $u=0$ согласно формуле Лейбница

$$
h^{(k)}(0)=\theta^{(k)}(0)+\sum_{s=1}^{k}\left(\begin{array}{l}
k-1 \\
s-1
\end{array}\right) \theta^{(s)}(0) h^{(k-s)}(0), \quad k=1,2, \ldots
$$

Поскольку

$$
h^{(k)}(0)=k ! c_{k}, \quad \theta^{(s)}(0)=s ! b_{s}, \quad s=0,1, \ldots, \quad k=0,1, \ldots,
$$

из этих равенств получаем (19).

Для вывода соотношений (21) используем дифференциальное уравнение (16). Так как

$$
\theta^{\prime}(u)=\sum_{r=0}^{\infty}(r+1) b_{r+1} u^{r}
$$

To

$$
\begin{aligned}
\alpha \theta^{\prime}(u) \theta(u) & =\alpha \sum_{r=0}^{\infty}(r+1) b_{r+1} u^{r} \sum_{s=0}^{\infty} b_{s} u^{s} \\
& =\sum_{k=0}^{\infty} \alpha \sum_{s=1}^{k+1} s b_{s} b_{k+1-s} u^{k}
\end{aligned}
$$

Далее,

$$
\begin{aligned}
\left(e^{\theta(u)}-1\right)\left(\theta^{\prime}(u)+2 u \theta(u)\right)= & \sum_{s=0}^{\infty} c_{s} u^{s}\left(b_{1}+\sum_{r=1}^{\infty}\left((r+1) b_{r+1}+2 b_{r-1}\right) u^{r}\right) \\
= & b_{1} c_{0}+\left(b_{1} c_{1}+2 c_{0}\left(b_{0}+b_{2}\right)\right) u \\
& +\sum_{k=2}^{\infty}\left(b_{1} c_{k}+\sum_{s=0}^{k-1} c_{s}\left((k-s+1) b_{k-s+1}+2 b_{k-s-1}\right)\right) u^{k} .
\end{aligned}
$$


Используя (16) и приравнивая коэффициенты при $u^{k}, k=2,3, \ldots$, получаем (21), для случаев $k=0$ и $k=1$ получаем тождества

$$
\begin{aligned}
c_{0} & =\alpha b_{0}, \\
\alpha b_{1}^{2} & =b_{1} c_{1}+2 b_{0} c_{0} .
\end{aligned}
$$

Теорема 2 доказана.

Следствие 1. При условиях теоремъ 1 равномерно относителъно $\alpha \in\left[\alpha_{0}, \alpha_{1}\right]$

$$
\begin{aligned}
|s(n, N)|= & \frac{n !}{N !} \sqrt{\frac{1-z_{0}}{2 \pi n\left(\alpha z_{0}-\alpha+1\right)}} \frac{\left(-\ln \left(1-z_{0}\right)\right)^{N}}{z_{0}^{n}} \\
& \times\left(1+\frac{f(\alpha)}{12 N\left(\alpha z_{0}-\alpha+1\right)^{3}}+O\left(N^{-2}\right)\right),
\end{aligned}
$$

гдe

$$
\begin{aligned}
f(\alpha)= & 6\left(\alpha z_{0}-\alpha+1\right)^{3}\left(\frac{1-z_{0}}{2 a_{0} z_{0}} \theta^{\prime \prime \prime}(0)-\frac{3 \alpha\left(1-z_{0}\right)}{2 z_{0}} \theta^{\prime \prime}(0)+\alpha^{2} a_{0}^{2}\right) \\
= & \alpha^{2}\left(1-z_{0}\right)^{2}\left(\alpha z_{0}-\alpha+3\right)+\left(4 \alpha-\frac{3}{2}\right) z_{0}^{2} \\
& +\left(3+\frac{1}{\alpha}\right) z_{0}+3-4 \alpha-\frac{1}{\alpha\left(1-z_{0}\right)} .
\end{aligned}
$$

Доказательство. Согласно (14) достаточно убедиться в том, что

$$
x_{1}=\frac{a_{2}}{a_{0}}=\frac{f(\alpha)}{6\left(\alpha z_{0}-\alpha+1\right)^{3}} .
$$

Для обоснования оценок (24) и (25), из которых следует (23), используем сначала (18), полагая $k=1$. При $k=1$ с учетом начальных условий (9) и (20) получаем, что

$$
a_{1}=\frac{2\left(1-z_{0}\right)}{z_{0}} b_{2}+\frac{2}{\alpha\left(\alpha z_{0}-\alpha+1\right)}
$$

Аналогично, при $k=2$ из (19) с учетом начальных условий (22) получаем, что

$$
c_{2}=\frac{b_{2}}{1-z_{0}}-\frac{z_{0}^{2}}{\alpha\left(1-z_{0}\right)^{2}\left(\alpha z_{0}-\alpha+1\right)} .
$$

Используя (21) при $k=2$, находим, что

$$
\frac{2-3 \alpha\left(1-z_{0}\right)}{1-z_{0}} b_{2}+c_{2}+\frac{2 z_{0}\left(\alpha\left(1-z_{0}\right)+1\right)}{\alpha\left(1-z_{0}\right)^{2}}=0 \text {. }
$$

Подставляя значение $c_{2}$ из (27), находим, что

$$
b_{2}=\frac{z_{0}\left(2 \alpha^{2}\left(1-z_{0}\right)^{2}+z_{0}-2\right)}{3 \alpha\left(1-z_{0}\right)\left(\alpha z_{0}-\alpha+1\right)^{2}}
$$


Если теперь использовать (18) при $k=2$ и учесть $(26),(27)$ и $(28)$, то получим, что

$$
\frac{a_{2}}{a_{0}}=\frac{3 b_{3}}{b_{1}}+\frac{2-2 \alpha\left(1-z_{0}\right)-z_{0}}{\left(\alpha z_{0}-\alpha+1\right)^{2}} .
$$

Далее, при $k=3$ из (19) следует, что

$$
c_{3}=b_{3}+\frac{1}{3}\left(b_{1} c_{2}+2 b_{2} c_{1}+3 b_{3} c_{0}\right)
$$

Используя соотношение (21) при $k=3$, подставляя в него найденное значение $c_{3}$ и деля обе части полученного равенства на $b_{1}^{2}$, после элементарных преобразований получаем, что

$$
\begin{aligned}
\frac{4\left(\alpha z_{0}-\alpha+1\right)}{1-z_{0}} \frac{b_{3}}{b_{1}} & +\frac{2 c_{1}}{b_{1}}\left(1+\frac{b_{2}}{3}\right) \\
& +\frac{2}{b_{1}^{2}}\left(\left(b_{0}+b_{2}\right) c_{2}+\alpha b_{2}\left(b_{0}-b_{2}\right)\right)+\frac{1}{3} c_{2}=0 .
\end{aligned}
$$

Выражая из (30) отношение $b_{3} / b_{1}$, подставляя значения $b_{2}$ и $c_{2}$ согласно (27) и (28) и используя (29), после элементарных выкладок получаем равенство (25), в котором величина $f(\alpha)$ определяется из (24). Тем самым оценка (23) доказана.

Оценка (23) дает неожиданно хорошее приближение уже при небольших значениях $n$ и $N$. Так, например, при $n=9$ и $N=6$ параметр $\alpha=3 / 2$ и $z_{0}=0,5335893 \ldots$ с точностью до $10^{-7}$, поэтому первое приближение, вычисленное по формуле (13) путем отбрасывания слагаемого порядка $O(1 / N)$, дает оценку 4688 (с точностью до целых). Второе приближение, вычисленное согласно (23) путем отбрасывания слагаемого порядка $O\left(1 / N^{2}\right)$, дает оценку 4530 вместо точного значения $|s(9,6)|=4536$ (см. [4], с. 61). При $n=12$ и $N=8$ получаем соответственно оценки 366416 и 357167 вместо точного значения $s(12,8)=357423$.

Заметим, что теорему 1 можно трактовать как теорему об асимптотических разложениях в области больших уклонений для числа циклов $\xi_{n}$ в подстановке степени $n$, выбираемой случайно и равновероятно из симметрической группы $S_{n}$, если исходить из известного равенства (см., например, [4], с. 87)

$$
\mathbf{P}\left\{\xi_{n}=N\right\}=\frac{|s(n, N)|}{n !}, \quad N=1,2, \ldots, n .
$$

\section{2. Числа Стирлинга второго рода}

В этом параграфе рассматривается задача асимптотической оценки чисел Стирлинга второго рода $\sigma(n, N)$, если $n, N \rightarrow \infty$ так, что выполнено условие (1). Как и для чисел Стирлинга первого рода $s(n, N)$, вопросам получения асимптотических оценок для чисел $\sigma(n, N)$ при различных предположениях о росте параметров $n, N$ посвящено к настоящему времени много работ (помимо работ, на которые имеются ссылки в [1], см. также [5-9]). В частности, в уже упоминавшейся работе Гуда [2], по-видимому, впервые получен главный член асимптотического разложения для чисел $\sigma(n, N)$ при условии $(1)$, а также выписаны рекуррентные формулы для 
коэффициентов при $N^{-1}$ и $N^{-2}$ в этом разложении. В этой же работе указывается, что в общем случае коэффициент при любой степени вида $N^{-m}, m=1,2, \ldots$, должен быть рациональной функцией от $\alpha=n / N$ и единственного вещественного положительного корня $z=z_{1}$ уравнения

$$
z\left(1-e^{-z}\right)^{-1}=\alpha>1 .
$$

Исходя из интегрального представления (см. [1])

$$
\sigma(n, N)=\frac{n !}{2 \pi i N !} \oint \frac{\left(e^{z}-1\right)^{N}}{z^{n+1}} d z
$$

(интегрирование в (32) осуществляется по окружности с центром в нуле, пробегаемой в положительном направлении), в этом параграфе показывается, что коэффициенты при отрицательных степенях вида $N^{-m}, m=1,2, \ldots$, в асимптотическом разложении чисел $\sigma(n, N)$ при условии (1) могут быть найдены, исходя из разложения в степенной ряд некоторой функции, выражающейся через решение нелинейного дифференциального уравнения первого порядка с известным начальным условием. Далее находятся линейные рекуррентные соотношения в комплексной области, которым удовлетворяют эти коэффициенты, и приводится явная формула для коэффициента при $N^{-1}$. Методы доказательства этих результатов, по существу, такие же, как и в предыдущем параграфе, поэтому в целях компактности изложения ограничимся лишь их точными формулировками.

Теорема 3. При условиях теоремы 1 равномерно относителъно $\alpha \in\left[\alpha_{0}, \alpha_{1}\right]$ справедливо асимптотическое разложение

$$
\sigma(n, N) \sim \frac{n !}{N !} \frac{z_{1}^{N-n}}{\left(\alpha-z_{1}\right)^{N} \sqrt{2 \pi n\left(1-\alpha+z_{1}\right)}}\left(1+\sum_{m=1}^{\infty} \frac{(2 m) !}{m ! 2^{2 m}} \frac{y_{m}}{N^{m}}\right),
$$

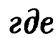

$$
y_{m}=\frac{A_{2 m}}{A_{0}}, \quad m=1,2, \ldots,
$$

и коэффициенты $A_{r}, r=0,1, \ldots$, определяотся из равенства

$$
\sum_{r=0}^{\infty} A_{r} u^{r}=\frac{\eta^{\prime}(u)}{\eta(u)}
$$

с началънъм условием

$$
A_{0}=i \sqrt{\frac{2}{\alpha\left(1-\alpha+z_{1}\right)}}
$$

В равенстве (34) функция $\eta(u)$ является аналитическим в окрестности нуля решением дифференциального уравнения

$$
\left(\alpha-\eta(u)\left(1-e^{-\eta(u)}\right)^{-1}\right) \eta^{\prime}(u)=2 u \eta(u),
$$

удовлетворяющим началъному условию

$$
\eta(0)=z_{1}
$$

При этом $z=z_{1}-$ единственный вещественный положителъный коренъ уравненuя (31). 
Теорема 4. Коэффициенты $A_{r}, r=0,1, \ldots$, в асимптотическом разложении (33) удовлетворяют линейным рекуррентным соотношениям в комплексной области

$$
\begin{aligned}
A_{r} & =\frac{1}{z_{1}}\left((r+1) C_{r+1}-\sum_{k=1}^{r} C_{k} A_{r-k}\right) \\
& =\frac{i\left(\alpha-z_{1}\right)}{z_{1}} \sqrt{\frac{\alpha}{2\left(1-\alpha+z_{1}\right)}}\left(\sum_{k=1}^{r}\left(B_{k+1}+\frac{C_{k+1}}{\alpha-z_{1}}\right) A_{r-k}-\frac{2}{\alpha} B_{r}\right), r=1,2, \ldots,
\end{aligned}
$$

с начальным условием (35). В (37) коэффициенты $B_{r}$ и $C_{r}, r=0,1, \ldots$, удовлетворяют рекуррентным соотночениям

$$
B_{r+1}=-\sum_{k=0}^{r}\left(1-\frac{k}{r+1}\right) B_{k} C_{r-k+1}, \quad r=0,1, \ldots,
$$

с началъными условиями

$$
\begin{aligned}
& B_{0}=1 \\
& C_{1}=A_{0} z_{1} \\
& C_{2}=\frac{z_{1}\left(\left(\alpha-z_{1}\right)\left(2 \alpha-z_{1}\right)-2\right)}{3 \alpha\left(1-\alpha+z_{1}\right)^{2}}
\end{aligned}
$$

Исходя из соотношений (33)-(38) и соответствующих начальных условий, получаем следующее утверждение.

Следствие 2. При условиях теоремы 1 равномерно относителъно $\alpha \in\left[\alpha_{0}, \alpha_{1}\right]$

$$
\sigma(n, N)=\frac{n !}{N !} \frac{z_{1}^{N-n}}{\left(\alpha-z_{1}\right)^{N} \sqrt{2 \pi n\left(1-\alpha+z_{1}\right)}}\left(1+\frac{g(\alpha)}{12 n\left(1-\alpha+z_{1}\right)^{3}}+O\left(n^{-2}\right)\right)
$$

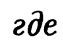

$$
\begin{aligned}
g(\alpha) & =6 \alpha\left(1-\alpha+z_{1}\right)^{3}\left(\frac{\eta^{\prime \prime \prime}(0)}{2 A_{0} z_{1}}-\frac{3 \eta^{\prime \prime}(0)}{2 z_{1}}+A_{0}^{2}\right) \\
& =-1+\left(\alpha-z_{1}\right)\left(z_{1}^{3}+\left(\frac{9}{2}-2 \alpha\right) z_{1}^{2}+\left(2 \alpha^{2}-9 \alpha+8\right) z_{1}-\alpha^{3}+3 \alpha^{2}-4 \alpha+3\right)
\end{aligned}
$$

Заметим, что оценки (39) и (40) дают очень хорошее приближение уже при небольших значениях $n$ и $N$. Так, например, при $n=4$ и $N=2$ параметр $\alpha=2$ и $z=1,593624 \ldots$ с точностью до $10^{-6}$ (значение $z_{1}$ заимствовано из [2]), поэтому первое приближение, вычисленное путем отбрасывания слагаемого $O\left(N^{-1}\right)$ в асимптотическом разложении (33), дает оценку 7,408; второе приближение, вычисленное по формулам (39) и (40) путем отбрасывания слагаемого порядка $O\left(n^{-2}\right)$ дает оценку 7,008 вместо точного значения $\sigma(4,2)=7$. При $n=10$ и $N=5$ первое и второе приближения дают соответственно оценки 43468 и 42531 (с точностью до целых) вместо точного значения $\sigma(10,5)=42525$ (см. [4]). Если же $n=100$ и $N=50$, то соответствующие оценки равны $4,31905 \cdot 10^{101}$ и $4,30974 \cdot 10^{101}$ (с точностью до 
пятого знака после запятой $)$, в то время как $\sigma(100,50) \approx 4,30983 \cdot 10^{101}$ с той же точностью (это значение заимствовано из [10]).

Пусть $\mu_{0}(n, N)$ - число ячеек, оказавшихся пустыми после бросания $n$ частиц в $N$ ячеек в классической задаче о размещении.

Следствие 3. Пусть $n, N \rightarrow \infty$ так, что

$$
1<\beta_{0} \leqslant \beta=\frac{n}{N-k} \leqslant \beta_{1}<\infty,
$$

где $k$ - челое неотричательное число (возможно, зависящее от $n u N$ ) $u \beta_{0}, \beta_{1}$ - постоянные. Тогда равномерно относителъно $\beta \in\left[\beta_{0}, \beta_{1}\right]$

$$
\begin{aligned}
\mathbf{P}\left\{\mu_{0}(n, N)=k\right\}= & \left(\begin{array}{c}
N \\
k
\end{array}\right) \frac{n !}{N^{n}} \frac{z_{2}^{N-n-k}}{\left(\beta-z_{2}\right)^{N-k} \sqrt{2 \pi n\left(1-\beta+z_{2}\right)}} \\
& \times\left(1+\frac{h(\beta)}{12 n\left(1-\beta+z_{2}\right)^{3}}+O\left(n^{-2}\right)\right),
\end{aligned}
$$

где $z=z_{2}-$ единственный вещественный положителъный коренъ уравнения (31), в котором в правой части равенства вместо $\alpha$ стоит $\beta$, и функция $h(\beta)$ определяется из (40) с заменой $g$ на $h, \alpha$ на $\beta$ и $z_{1}$ на $z_{2}$.

Оценка (41) следует из (39) и (40) и известного равенства

$$
\mathbf{P}\left\{\mu_{0}(n, N)=k\right\}=\frac{N !}{k ! N^{n}} \sigma(n, N-k),
$$

справедливого при $k=0,1, \ldots, N$. Эта оценка дает высокую точность приближения даже при небольших значениях $n$ и $N$. Если, например, $n=N=10$ и $k=5$, то $\beta=2$ и $z_{2}=1,593624 \ldots$, и вычисления по формуле (41) с отбрасыванием слагаемого порядка $O\left(n^{-2}\right)$ дают оценку 0,12861 (с точностью до $10^{-5}$ ) вместо точного значения $\mathbf{P}\left\{\mu_{0}(10,10)=5\right\}=0,1285956$. В то же время, если положить $\lambda=\mathbf{E} \mu_{0}(10,10) \approx 3,48678$ (с той же точностью), то известная предельная теорема Пуассона в правой области для случайной величины $\mu_{0}(n, N)$ дает оценку $\lambda^{5} e^{-\lambda} / 5 ! \approx 0,13142$; эта оценка в данном случае практически совпадает со значением главного члена в формуле (41), равным 0,13145 .

\section{Список литературы}

1. Сачков В. Н. Комбинаторные методы дискретной математики. Наука, Москва, 1977.

2. Good I. J. An asymptotic formula for the differences of the powers at zero. Ann. Math. Statist. (1961) 32, №1, 249-256.

3. Евграфов М. А. Асимтотические оценки и челые функции. Физматлит, Москва, 1962.

4. Риордан Дж. Введение в комбинаторный анализ. Изд-во иностр. лит., Москва, 1963.

5. Hsu L. C. Note on an asymptotic expansion of the $n$th difference of zero. Ann. Math. Statist. (1948) 19, 273-277.

6. Arfwedson G. A probability distribution connected with Stirling's second-class numbers. Skand. Aktuarietidskrift (1951) 34, 121-132.

7. Bender E. A. Asymptotic methods in enumerations. SIAM Rev. (1974) 16, 485-515. 
8. Gould H. W. Euler's formula for $n$th differences of powers. Amer. Math. Monthly (1978) $85,450-467$.

9. Holst L. On numbers related to partition of unlike objects and occupancy problems. European J. Combinatorics (1981) 2, 231-237.

10. Bleick W. E., Wang P. C. C. Asymptotic of Stirling numbers of the second kind. Proc. Amer. Math. Soc. (1974) 42, №2, 575-580.

Статья поступила 21.04.1997. Переработанный вариант поступил 18.05.1998. 